Jeremy M. Kahn

\title{
The evolving role of dedicated weaning facilities in critical care
}

Received: 1 September 2009

Accepted: 3 September 2009

Published online: 26 September 2009

(C) Copyright jointly hold by Springer and ESICM 2009

This editorial refers to the article available at:

doi:10.1007/s00134-009-1658-2.

\section{J. M. Kahn (区)}

Division of Pulmonary, Allergy and Critical Care,

Center for Clinical Epidemiology and Biostatistics,

University of Pennsylvania School of Medicine, Blockley Hall 723, 423 Guardian Drive, Philadelphia, PA 19104, USA

e-mail: jmkahn@mail.med.upenn.edu

Tel.: +1-215-5737791

J. M. Kahn

Leonard Davis Institute of Health Economics,

University of Pennsylvania, Philadelphia, PA, USA

Many patients with critical illness survive their acute episode yet have persistent respiratory failure requiring prolonged mechanical ventilation (PMV) [1]. PMV is an extraordinarily large clinical and financial burden on the health system. In the United States, over 100,000 patients require PMV each year, and this number is expected to rise as the population ages and demand for critical care increases [2]. PMV patients already consume a disproportionate amount of health care resources; although they make up less than $10 \%$ of all ICU patients, they account for $40 \%$ of ICU expenditures [3]. Less than half of PMV patients are alive 1 year after hospital discharge, and survivors frequently experience significant reductions in health-related quality of life [4-6].

Traditionally patients with PMV received all of their acute care in an ICU. However, over the last 20 years the care of patients with PMV evolved to include facilities dedicated purely to weaning. These types of facilities go by several names, including step-down units, respiratory intensive care units, and intermediate care units, among others. Dedicated weaning facilities typically distinguish themselves from ICUs by containing less intense clinician staffing, protocols for long-term ventilator care, and physiotherapists with expertise in this area [7]. For the most part these facilities operate as separate units within hospitals [8]. In the United States, however, a new type of weaning facility recently emerged-long-term acute care hospitals (LTACs). LTACs are like step-down units but exist as completely independent hospitals [9]. Driven by increasing demand and financial incentives that favor inter-hospital transfers, LTACs are the fasting growing segment of acute care in the United States, expanding at a rate of $12 \%$ per year [10]. Currently there is wide international variation in access to dedicated weaning facilities - Canada and the United Kingdom have nearly none, while other European countries and the U.S. have a significant number.

Dedicated weaning facilities such as LTACs and intermediate care units have the potential to offer unique benefits to patients with PMV. Compared to traditional ICUs, they may improve survival through increased clinical experience in chronic respiratory failure, use of specialized multidisciplinary teams, and advanced weaning protocols [11]. Weaning facilities also may reduce health care costs, as they are generally less costly than ICUs on a per-day basis and may speed weaning compared to ICUs. The benefits of dedicated weaning facilities may also extend beyond the patients they admit. ICU beds are a limited resource-by admitting patients with long ICU stays, weaning facilities can unburden ICUs, resulting in an effective increase in the number of ICU beds, which may benefit society as a whole.

Yet these gains in quality and efficiency are not assured. Dedicated weaning facilities could actually 
worsen survival by fragmenting care, disrupting communication, and providing lower nurse-to-patient ratios than traditional ICUs. Dedicated weaning facilities may also increase overall costs, either by paradoxically delaying weaning or inducing a tolerance for PMV. Physicians may wean more slowly without the pressures of constrained bed supply or may be less willing to have difficult end-of-life discussions with families if they know they can simply transfer patients to weaning facilities instead of withdrawing life support.

A diverse program of comparative effectiveness research is needed to better understand these risks and benefits. Unfortunately, to date only a few studies have examined the clinical and economic effects of transfering to dedicated weaning facilities compared to remaining in an ICU for patients with PMV [10, 12]. These studies generally show that dedicated weaning facilities are cost saving from the perspective of the hospital but have little impact on overall costs or survival. No studies exist comparing costs and outcomes between the different types of dedicated weaning facilities, such as those within hospitals and those that operate as stand-alone hospitals. Nor is there any information on the relationship between the organizational attributes of weaning facilities and outcomes. An extensive body of literature shows that intensivist physician staffing, low nurse-to-patient ratios, multidisciplinary care teams and care protocols are beneficial in the ICU, yet no analogous literature exists for weaning facilities [13].

In this issue of Intensive Care Medicine, Polverino and colleagues take a first step toward filling this knowledge gap [14]. They examine patient characteristics, outcomes, and practice patterns in five Italian respiratory intensive care units from 1991 to 2005 . Over the 15 -year period, the number of beds and admissions per year markedly increased, mirroring similar findings in the U.S. health system. Severity of illness and the number of co-morbid conditions also increased over time. Outcomes generally worsened, with survival, weaning success, and proportion of patients transferred home without respiratory support all decreasing over time, coincident with increases in the physician-to-patient staffing ratio. Although it is possible that the worsened outcomes are due to changes in weaning unit organization, a much more compelling scenario is that disease severity increased over the years, as the care of patients with chronic critical illness increasingly shifted from ICUs to specialized weaning units.

These results should be interpreted with caution. The authors had only hospital-level data, and thus could not perform a multivariate analysis to identify factors independently associated with outcomes. Also, the findings are subject to the ecological fallacy, an epidemiological phenomenon that occurs when population-level data are used to make inferences at the individual level [15]. Thus relationships that the authors observed at the unit level may not be true for individual patients. Yet this study provides preliminary empirical evidence of a link between organization and outcome in dedicated weaning facilities and reminds us of the pressing need to rigorously study the structural factors relating to the outcome of patients with PMV.

The number of dedicated weaning facilities is likely to increase in the coming years, and the role for these facilities will continue to evolve. As efforts to reduce health care costs shift patients away from ICUs toward other clinical settings, dedicated weaning facilities will care for both more and increasingly complex patients. It is essential that we critically evaluate these changes as they occur. More research is needed on the impact of weaning facilities on costs and outcomes; whether weaning facilities best operate as units within hospitals or as standalone hospitals; the optimum nurse, physician, and ancillary staffing of weaning facilities; and the spillover effects of weaning facilities on critical care. Just as we seek evidence to inform the process of care for patients with PMV, the organization and structure of care for these patients must also be evidence-based.

\section{References}

1. MacIntyre NR, Epstein SK, Carson S, Scheinhorn D, Christopher K, Muldoon S (2005) Management of patients requiring prolonged mechanical ventilation: report of a NAMDRC consensus conference. Chest 128:39373954

2. Carson SS, Bach PB (2002) The epidemiology and costs of chronic critical illness. Crit Care Clin 18:461476

3. Wagner DP (1989) Economics of prolonged mechanical ventilation. Am Rev Respir Dis 140:S14-S18
4. Schonhofer B, Euteneuer S, Nava S, Suchi S, Kohler D (2002) Survival of mechanically ventilated patients admitted to a specialised weaning centre. Intensive Care Med 28:908-916

5. Cox CE, Carson SS, Lindquist JH, Olsen MK, Govert JA, Chelluri L (2007) Differences in one-year health outcomes and resource utilization by definition of prolonged mechanical ventilation: a prospective cohort study. Crit Care 11:R9
6. Pilcher DV, Bailey MJ, Treacher DF, Hamid S, Williams AJ, Davidson AC (2005) Outcomes, cost and long term survival of patients referred to a regional weaning centre. Thorax 60:187-192

7. Nava S, Confalonieri M, Rampulla C (1998) Intermediate respiratory intensive care units in Europe: a European perspective. Thorax 53:798-802 
8. Corrado A, Roussos C, Ambrosino N, Confalonieri M, Cuvelier A, Elliott M, Ferrer M, Gorini M, Gurkan O, Muir JF, Quareni L, Robert D, Rodenstein D, Rossi A, Schoenhofer B, Simonds AK, Strom K, Torres A, Zakynthinos S (2002) Respiratory intermediate care units: a European survey. Eur Respir J 20:1343-1350

9. Scheinhorn DJ, Hassenpflug MS, Votto JJ, Chao DC, Epstein SK, Doig GS, Knight EB, Petrak RA (2007) Post-ICU mechanical ventilation at 23 long-term care hospitals: a multicenter outcomes study. Chest 131:85-93
10. Medicare Payment Advisory

Commission (2004) Defining long term acute care hospitals. In: Report to

Congress: new approaches in Medicare. Washington DC

11. Subbe CP, Criner GJ, Baudouin SV (2007) Weaning units: lessons from North America? Anaesthesia 62:374-380

12. Seneff MG, Wagner D, Thompson D, Honeycutt C, Silver MR (2000) The impact of long-term acute-care facilities on the outcome and cost of care for patients undergoing prolonged mechanical ventilation. Crit Care Med 28:342-350
13. Carmel S, Rowan K (2001) Variation in intensive care unit outcomes: a search for the evidence on organizational factors. Curr Opin Crit Care 7:284-296

14. Polverino E, Nava S, Frerrer M, Ceriana P, Clini E, Spada E, Zanotti E, Trianni L, Barbano L, Fracchia C, Vitacca M, Balbi B (2009) Patient characterization, hospital course and clinical outcomes in five Italian respiratory intensive care units. Intensive Care Med. doi: 10.1007/s00134-009-1658-2

15. Greenland S, Robins J (1994) Ecologic studies-biases, misconceptions, and counterexamples. Am J Epidemiol 139:747-760 